\title{
Cost-Effectiveness of Transcatheter Aortic Valve Implantation in Intermediate and Low Risk Severe Aortic Stenosis Patients in Singapore
}

Ivandito Kuntjoro, ${ }^{1} M D$, Edgar $\underline{\text { Tay, }}{ }^{1} M B B S$, Jimmy $\underline{\text { Hon }},{ }^{2} M B C h B$, James Yip,${ }^{1} M B B S$, William Kong, ${ }^{1} M B C h B$, Kian Keong $\underline{\text { Poh }},{ }^{1}$ MBBChir, Tiong Cheng $\underline{\text { Yeo }},{ }^{1}{ }_{M B B S}$, Huay Cheem $\underline{\text { Tan }},{ }^{1}{ }_{M B B S}$, Michael George Caleb,${ }^{2}{ }_{M B B S}$, Nan Luo, ${ }^{3}$ PhD, MSc ; Pei Wang, ${ }^{4,5}$ PhD

\begin{abstract}
Introduction: Singapore has the world's second most efficient healthcare system while costing less than 5\% GDP. It remains unclear whether transcatheter aortic valve implantation (TAVI) is cost-effective for treating intermediate-low risk severe aortic stenosis (AS) patients in a highly efficient healthcare system. Materials and Methods: A two-phase economic model combining decision tree and Markov model was developed to assess the costs, effectiveness, and the incremental cost-effectiveness ratio (ICER) of transfemoral (TF) TAVI versus surgical aortic valve replacement (SAVR) in intermediate-low risk patients over an 8-year time horizon. Mortality and complications rates were based on PARTNER 2 trial cohort $A$ and Singapore life table. Costs were mainly retrieved from Singapore National University Health System database. Health utility data were obtained from Singapore population based on the EuroQol-5D (EQ-5D). A variety of sensitivity analyses were conducted. Results: In base case scenario, the incremental effectiveness of TF-TAVI versus SAVR was 0.19 QALYs. The ICER of TF-TAVI was S\$33,833/QALY. When time horizon was reduced to 5 years, the ICER was $\$ \$ 60,825 /$ QALY; when event rates from the propensity analysis was used, the ICER was S\$21,732/QALY and S\$44,598/QALY over 8-year and 5-year time horizons, respectively. At a willingness to pay threshold of $\$ \$ 73,167 /$ QALY, TF-TAVI had a $98.19 \%$ probability of being cost-effective after 100,000 simulations. The model was the most sensitive to the costs of TF-TAVI procedure. Conclusion: TF-TAVI is a highly cost-effective option compared to SAVR for intermediate-low risk severe AS patients from a Singapore healthcare system perspective. Increased procedure experience, reduction in device cost, and technology advance may have further increased the cost-effectiveness of TF-TAVI per scenario analysis.
\end{abstract}

Ann Acad Med Singapore 2020;49:423-33

Key Words: Surgical aortic valve replacement, Quality of life, Transfemoral

TAVI, Reimbursement

\section{Introduction}

Transcatheter aortic valve implantation (TAVI) has been shown to be effective in treating patients with severe symptomatic aortic stenosis (AS) who are in the intermediate, high and prohibitive risk for conventional surgical aortic valve replacement (SAVR). ${ }^{1-5}$ Several cost-effectiveness analyses have been conducted for this therapy in comparison to patients undertaking SAVR. ${ }^{6-14}$ Although there were some heterogeneous outcomes in the early years of TAVI, ${ }^{6,8,9,13,14}$ recent analyses are consistently showing that TAVI is a cost-effective therapy. ${ }^{7,10,11}$ This is the result of improvements made to the newer iterations of TAVI devices, better patient selection and overcoming the learning curve of the procedure. ${ }^{15-18}$ This leads to better clinical outcomes, less re-hospitalisations, and shorter length of stay and thus overall resource utilisation. ${ }^{16,17}$

\footnotetext{
${ }^{1}$ Department of Cardiology, National University Heart Centre, Singapore

${ }^{2}$ Department of Cardiac, Thoracic and Vascular Surgery, National University Heart Centre, Singapore

${ }^{3}$ Saw Swee Hock School of Public Health, National University of Singapore, Singapore

${ }^{4}$ School of Public Health, Fudan University, Shanghai, China

${ }^{5}$ Key Lab of Health Technology Assessment, National Health Commission of the People's Republic of China (Fudan University), Shanghai, China

Address for Correspondence: A/Prof Wang Pei, School of Public Health, Fudan University, 130 Dong An Road, Shanghai, 200032, China

Email: Wang_p@fudan.edu.cn
} 
The adoption of TAVI is widespread among the Western world (i.e. the United States and Europe).${ }^{19}$ This is due to positive publications on clinical outcomes and cost-effective analyses in these countries, which had subsequently led to national reimbursement. Many of these healthcare systems, however, spend a significant portion of their GDP on healthcare. ${ }^{20}$ Singapore is ranked the world's second most efficient healthcare system by the Bloomberg Health Care Efficiency Index, while remaining one of the lowest in percentage of GDP spent on healthcare. ${ }^{20,21}$ The hybrid (public and private) healthcare services and insurance model enables competition on costs and quality of services. As the cost of healthcare services and patients' quality of life for the same disease vary in different healthcare systems, it is important to understand whether TAVI remains cost-effective in a lower healthcare spending environment. The outcomes of this study may have a direct impact on long term funding in the country, and potentially on funding in countries or regions with a similar healthcare structure.

Considering transfemoral (TF) access is the predominant TAVI route used in Singapore, we aim to assess the cost-effectiveness of TF-TAVI versus SAVR in intermediate-low risk patients with severe AS, based on clinical results from PARTNER 2A trial (or PARTNER 2 S3 observational trial), as well as hospital costs and clinical practice data from Singapore.

\section{Materials and Methods}

We adopted a 2-phase economic model to assess the cost-effectiveness of 2 treatment options: TF-TAVI versus SAVR from the perspective of the National University Health System (NUHS) in Singapore. Outcomes were expressed as quality-adjusted life-years (QALYs) gained. We used a pre-defined willingnessto-pay threshold value of S\$73,167 (US\$54,198) per QALY, based on per capita GDP of Singapore in 2017, as indicated by the World Health Organization. ${ }^{22}$ Hence, the new therapy would be considered costeffective if its additional costs required to generate one QALY is below the threshold. The model development and analyses were performed using TreeAge Pro Suite 2013 (TreeAge Software, Inc, Williamstown, MA).

\section{Time Horizon}

The starting age in the model is 82 years for intermediate to low risk patients to match the average age of the PARTNER 2A trial (standard deviation: 6.7 years old), ${ }^{2,23}$ which is consistent with the average age (81 years old, standard deviation 7.9) of patients of the same risk level who received TF-TAVI at
NUHS. The life expectancy for local residents at age 82 is about 8 years according to Singapore life ables. ${ }^{24}$ Hence, the time horizon was set as 8 years.

\section{Model Structure}

Short-term phase: A decision tree model was constructed to estimate the expected costs and QALYs of the 2 different treatment modalities for the initial 30 days after procedure. Patients during this period are at risks of operative death and postoperative complications. Postoperative complications can be acute or chronic. Acute complications including endocarditis, vascular complications, paravalvular leak (PVL), pacemaker implantation, major bleeding, and atrial fibrillation are assumed to only impact short-term costs and QALYs. Chronic complications including stroke, acute kidney injury (AKI), and myocardial infarction (MI) are assumed to have a longer impact. In addition, patients may also die from any of the complications (Fig. 1A).

Long-term phase: The long-term costs and effectiveness beyond 30 days after procedure were estimated using a Markov model (Fig. 1B) with annual cycles. Patients surviving in the short-term model without complications or experiencing acute complications enter the "Alive without complications' health state in the long-term model. Patients surviving a chronic complication in the short-term model enter the long-term model in their respective health states. At the end of each cycle, patients transition among these states according to his/her current status and the model will run again. We assume patients with chronic complications stay in those health states until they died.

\section{Clinical Parameters}

Postoperative mortality rates for patients receiving TF-TAVI and SAVR treatments were derived from the PARTNER 2A trial. Yearly mortality rates within the maximum reported follow up duration were estimated through calibration of the results of the trials. To avoid double counting, the number of patients dying at 30 days, 1 year, or 2 years were removed from mortality estimation at 1 year or 2 years. We also estimated annual mortality rates between $2-5$ years according to the 5-year cumulative mortality rate by assuming linear increase over time, ${ }^{25}$ and derived the rates after 5 years based on the age-adjusted mortality rate of the general Singaporean population. ${ }^{24}$ Compared with the general population, the mortality rates for patients without or with chronic complications were assumed as the same or as a 2-fold increase, reflecting the higher mortality burden after the event, respectively. ${ }^{26,27}$ 
The 30-day postoperative complication rates and the proportion requiring additional pacemakers were derived from the trial (Table 1). Complications such as PVL are assumed as an acute complication in the short-term phase (Fig. 1A) due to 2 reasons. First, severe PVL is rare and mostly occur during post-procedure inpatient period $(0.3 \%$ for both TAVI and SAVR cohort at 30-day). ${ }^{2}$ Second, although the prevalence of moderate or more PVL prevalence is higher in TAVR than in SAVR $(3.7 \%$ vs $0.5 \%),{ }^{2}$ and PVL may affect longer term mortality, ${ }^{25}$ we omitted it in the Markov model due to data unavailability (i.e., costs and health utility values for PVL) and the consideration of a parsimonious model. ${ }^{28}$ Event rates for chronic complications at the first and second year were also obtained from the cohort. As there was no significant difference in the events after 2 years between TF-TAVI and SAVR, we assumed that the 2 treatments had the same rate for each chronic complication after 2 years. Each rate was calculated as the mean of corresponding rates from the 2 treatments at 2 years, and will remain constant for the remaining years. We also excluded re-hospitalisations and re-interventions after TAVI because of the similar reasons of dropping PVL, albeit their prevalence in the TAVI arm was higher at 5 years. ${ }^{25}$

\section{Costs}

Only direct medical (and unsubsidised) costs were included in the analysis. The index hospitalisation and one-year hospital follow-up costs were retrieved from the NUHS database. The index hospitalisation costs included were pre-procedural costs, procedural costs (including device cost), and post-procedural costs. A cost of $S \$ 40,000$ for the TAVI valve system and the median cost of $\mathrm{S} \$ 4,500$ for surgical bioprosthesis valve were used. Costs were calculated in Singapore dollars in 2017, and future costs were discounted at an annual rate of $3 \%$. Costs related to chronic complications are based on data published from NUHS (i.e. stroke and MI ${ }^{29}$ or the National Kidney Foundation (i.e. AKI), the main hemodialysis services provider in Singapore. ${ }^{30}$ Based on these publications, costs within the first year post onset of chronic complication includes costs for emergency room, doctor consultation, procedure and hospitalisation. Costs in subsequent years include medication costs, follow-up outpatient visits and laboratory tests, assuming 4 visits in a year, as well as pacemaker annual checkup costs. Costs are summarised in Table 2.

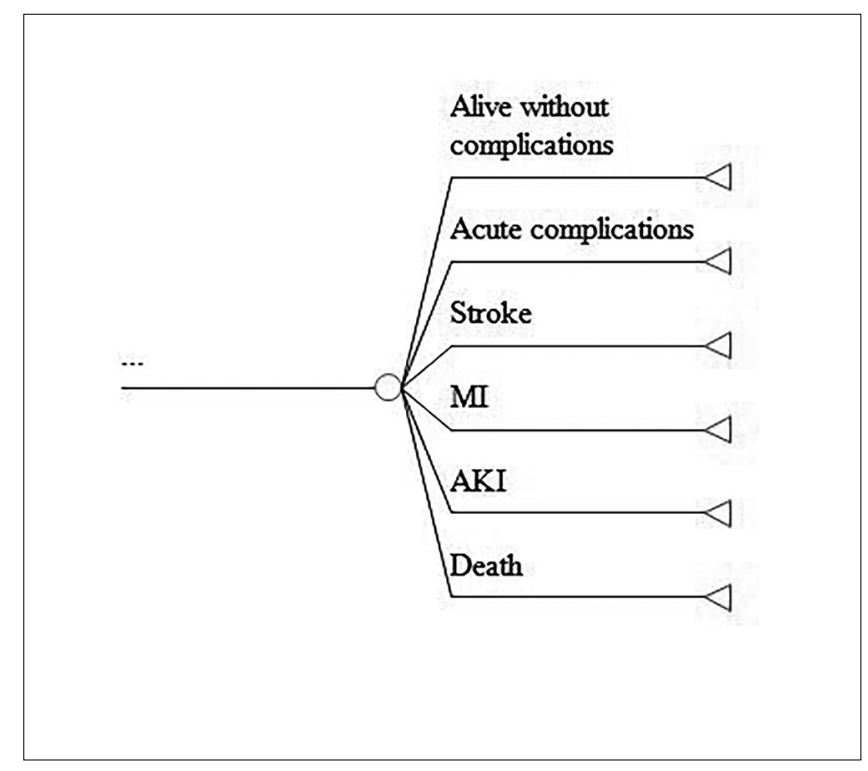

Fig. 1A. Two-phase Economic Model Structure: Structure of Short-term Decision Tree.

AKI: Acute kidney injury; MI: Myocardial infarction

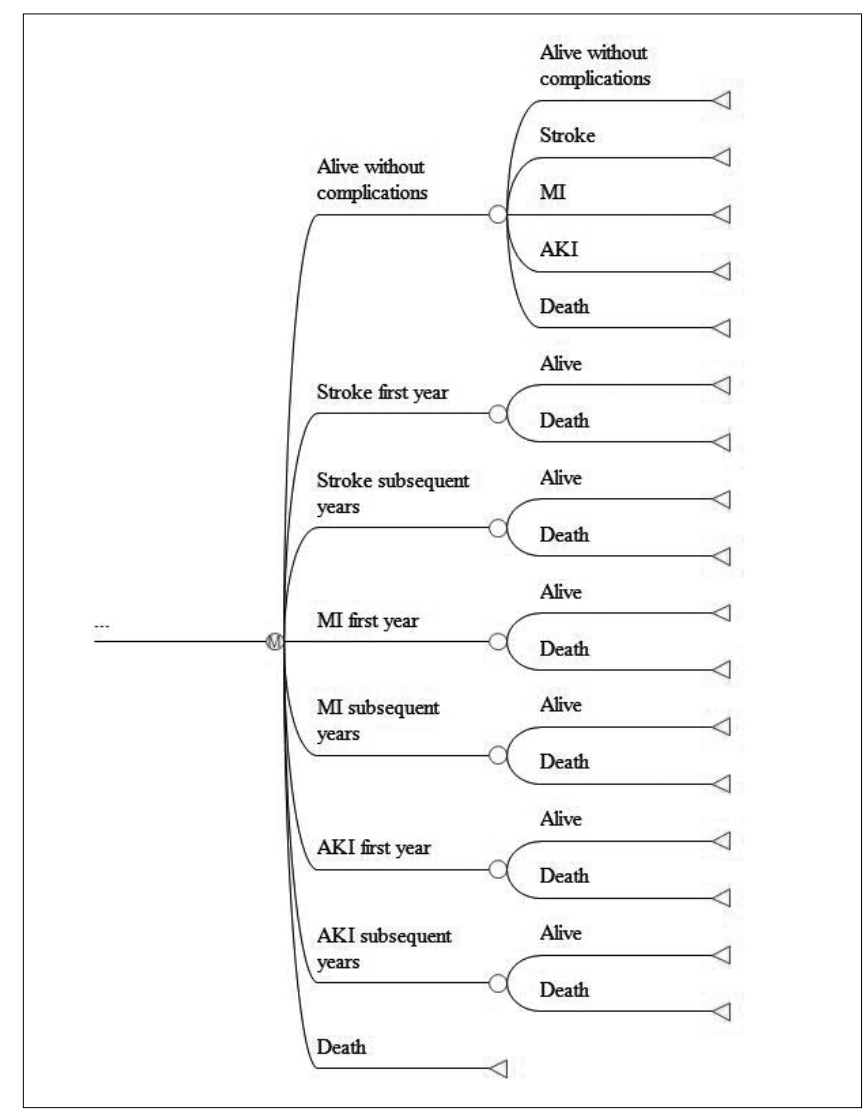

Fig. 1B. Two-phase Economic Model Structure: Structure of Long-term Markov Model.

AKI: Acute kidney injury; MI: Myocardial infarction 
Table 1. Event Probabilities

\begin{tabular}{|c|c|c|c|c|}
\hline & TF-TAVI $\dagger$ & SAVR $\dagger$ & TF-TAVI & SAVR \\
\hline \multicolumn{5}{|l|}{ Postoperative outcomes } \\
\hline Postoperative death & 3.0 & 4.1 & 1.1 & 3.9 \\
\hline Atrial fibrillation & 4.9 & 5.9 & 3.2 & 28.5 \\
\hline Endocarditis & 0 & 0 & 0.2 & 0 \\
\hline Life threatening/disabling bleeding & 6.7 & 41.4 & 3.6 & 44.2 \\
\hline Pacemaker implantation & 8.1 & 7.1 & 10.5 & 7.6 \\
\hline Major vascular complications & 8.5 & 3.9 & 6.4 & 4.2 \\
\hline Stroke & 4.2 & 6.3 & 2.5 & 6.3 \\
\hline MI & 0.6 & 1.8 & 0.3 & 1.8 \\
\hline AKI & 0.5 & 3.0 & 0.5 & 3.3 \\
\hline \multicolumn{5}{|l|}{ Long-term outcomes } \\
\hline \multicolumn{5}{|l|}{ Mortality } \\
\hline 1 year & 5.9 & 7.9 & 5.5 & 8.6 \\
\hline 2 year & 4.4 & 5.0 & LT & LT \\
\hline 5 year & $42.7 *$ & $40.5^{*}$ & LT & LT \\
\hline After 5 years & LT & LT & LT & LT \\
\hline $\begin{array}{l}\text { Stroke } \\
1 \text { year } \\
2 \text { year }\end{array}$ & $\begin{array}{l}2.7 \\
1.4\end{array}$ & $\begin{array}{l}2.0 \\
0.6\end{array}$ & $\begin{array}{l}1.8 \\
\text { NA }\end{array}$ & $\begin{array}{l}2.2 \\
\text { NA }\end{array}$ \\
\hline $\begin{array}{l}\text { MI } \\
1 \text { year } \\
2 \text { year }\end{array}$ & $\begin{array}{l}1.3 \\
1.0\end{array}$ & $\begin{array}{l}1.2 \\
0.9\end{array}$ & $\begin{array}{l}1.5 \\
\text { NA }\end{array}$ & $\begin{array}{l}0.9 \\
\text { NA }\end{array}$ \\
\hline $\begin{array}{l}\mathbf{A K I} \\
1 \text { year } \\
2 \text { year }\end{array}$ & $\begin{array}{l}1.6 \\
0.3\end{array}$ & $\begin{array}{l}2.0 \\
1.0\end{array}$ & $\begin{array}{l}\text { NA } \\
\text { NA }\end{array}$ & $\begin{array}{l}\text { NA } \\
\text { NA }\end{array}$ \\
\hline
\end{tabular}

AKI: Acute kidney injury; LT: Singapore life table; MI: Myocardial infarction; NA: Not applicable; SAVR: Surgical aortic valve replacement; TF-TAVI: Transfemoral transcatheter aortic valve replacement

* cumulative mortality rate.

†probabilities from the PARTNER 2A RCT.

f probabilities from the PARTNER 2 S3 observational trial.

\section{Qaly and Health Utilities}

QALY was calculated by multiplying the time duration in each health state with the quality-of-life weight for the state. The weights in the QALY approach are a set of health utilities. Baseline age and gender adjusted health utilities and chronic complications (i.e. stroke, MI, and AKI) were taken from the Singapore population norm for EuroQol-5D (EQ-5D) using local preference weights (Table 3 ). ${ }^{31}$ The utility of the atrial fibrillation (AF) was derived from a local study. ${ }^{32}$ The utilities of the other acute complications were deemed equivalent to that of AF since local utilities for the complications have not been established. The QALYs after 1 year was discounted at an annual rate of $3 \%$.

\section{Base Case Analysis}

The estimated values of model parameters combined with the assumptions of costs and effectiveness made above were used to calculate the incremental costeffectiveness ratio (ICER) between TF-TAVI and SAVR over the 8-year horizon. 
Table 2. Standardised Costs at Index Admission and Long-term Costs

\begin{tabular}{|c|c|c|c|c|}
\hline & TF-TAVI & & SAVR & Reference \\
\hline Pre-procedural costs, S\$ & & & & NUHS \\
\hline Transthoracic echocardiogram & & 168 & & \\
\hline Coronary angiogram & & 1,669 & & \\
\hline CT thorax & & 929 & & \\
\hline Ultrasound carotids/lung function test & & 183 & & \\
\hline Overall & & 2,949 & & \\
\hline Procedural costs, $\mathrm{S} \$$ & & & & NUHS \\
\hline Depreciation & 513 & & 1,046 & \\
\hline Maintenance & 329 & & 437 & \\
\hline Consumables & 1,156 & & 4,166 & \\
\hline Drugs & 40 & & 33 & \\
\hline Manpower & 3,807 & & 6,629 & \\
\hline Overheads & 1,871 & & 3,940 & \\
\hline Facility fees & 7,716 & & 16,251 & \\
\hline Implant & 40,000 & & 4,500 & \\
\hline Overall & 55,432 & & 37,002 & \\
\hline Post-procedural costs, $\mathrm{S} \$$ & 8,000 & & 16,000 & NUHS \\
\hline Cost of pacemaker (if any), S\$ & & & & NUHS \\
\hline Pacemaker implantation (B2 ward) & & 3,200 & & \\
\hline Pacemaker device (median) & & 15,000 & & \\
\hline First year pacemaker check (standard twice a year) & & 122 & & \\
\hline One year follow-up costs, S\$ & & & & NUHS \\
\hline Physician consultation & 295 & & 591 & \\
\hline Lab tests & 130 & & 338 & \\
\hline Radiology and electrocardiogram & 104 & & 104 & \\
\hline Portable cardiac echo-Doppler & 413 & & 413 & \\
\hline Medication & 46 & & 48 & \\
\hline Overall & 988 & & 1,494 & \\
\hline \multicolumn{5}{|l|}{ Costs of complications, $\mathbf{S \$}$} \\
\hline After stroke first year & & 14,000 & & Lin $2015^{29}$ \\
\hline After MI first year & & 12,354 & & Lin $2015^{29}$ \\
\hline After AKI first year & & 16,823 & & Yang $2016^{30}$ \\
\hline After stroke subsequent years & & 1,070 & & Lin $2015^{29}$ \\
\hline After MI subsequent years & & 1,214 & & Lin $2015^{29}$ \\
\hline After AKI subsequent years & & 26,179 & & Yang $2016^{30}$ \\
\hline
\end{tabular}

AKI: Acute kidney injury; CT: Computed tomography; MI: Myocardial infarction; NUHS: National University Hospital System, Singapore; SAVR: Surgical aortic valve replacement; TF-TAVI: Transfemoral transcatheter aortic valve replacement

* Lin L, Teng M, Zhao YJ, Khoo AL, Seet RC, Yong QW, et al. Long-term cost-effectiveness of statin treatment for primary prevention of cardiovascular disease in the elderly. Cardiovasc Drugs Ther 2015;29:187-97.

$\dagger$ Yang F, Lau T, Luo N. Cost-effectiveness of haemodialysis and peritoneal dialysis for patients with end-stage renal disease in Singapore. Nephrology (Carlton) 2016;21:669-77. 
Table 3. Health Utility Scores and its References

\begin{tabular}{lcc}
\hline & Utility & Reference \\
\hline Baseline & 0.78 & Abdin $2015^{31}$ \\
Alive without complications & 0.88 & Abdin $2015^{31}$ \\
Acute complications & 0.86 & Wang $2014^{32}$ \\
& Disutility (95\% CI) & Abdin $2015^{31}$ \\
\hline Stroke & $-0.24(-0.40,-0.07)$ & Abdin $2015^{31}$ \\
MI & $-0.1(-0.15,-0.05)$ & Abdin $2015^{31}$ \\
\hline AKI & $-0.13(-0.26,-0.01)$ & \\
\hline
\end{tabular}

AKI: Acute kidney injury; CI: confidence interval; MI: Myocardial infarction

* Abdin E, Subramaniam M, Vaingankar JA, Luo N, Chong SA. Population norms for the EQ-5D index scores using Singapore preference weights. Qual Life Res 2015;24:1545-53.

$\dagger$ Wang Y, Xie F, Kong MC, Lee LH, Ng HJ, Ko Y. Cost-effectiveness of dabigatran and rivaroxaban compared with warfarin for stroke prevention in patients with atrial fibrillation. Cardiovasc Drugs Ther 2014;28:575-85.

\section{Sensitivity and Scenario Analyses}

The impact of uncertainty around the model's parameters on the cost-effectiveness results was assessed using scenario and sensitivity analyses. Specifically, scenario analyses were used to explore the sensitivity of results to alternative model assumptions and parameter values chosen. For instance, we adopted the events rates from PARTNER 2 S3 propensity analysis that used newer TAVI systems. A range of one-way deterministic sensitivity analyses (DSA) were performed to determine how key model parameters would affect the results. We assumed all costs varied within a range of $75 \%$ to $125 \%$ of their respective values; the ranges of disutility values were based on their respective $95 \%$ confidence intervals; and the discount rates for both costs and QALY ranged from $1 \%$ to $6 \%$. Probabilistic sensitivity analysis was conducted to simultaneously assess the impact of uncertainty of all model parameters on results, using a Monte Carlo simulation with 100,000 iterations with random draws from each parameter distribution. We assumed beta distributions for clinical events rates, transition probabilities, and health utilities; and a gamma distribution for costs. Costeffectiveness acceptability curve was used to express the decision uncertainty (i.e. the probability that TF-TAVI is cost-effective) given a range of willingness to pay threshold values.

\section{Results}

\section{Base-Case Analysis}

Based on the clinical outcomes of the TF cohort, TF-TAVI generated QALY gains of 3.86 and was associated with expected costs of $\mathrm{S} \$ 75,386$ in comparison with 3.67 and $\mathrm{S} \$ 69,140$ generated by SAVR over 8 years (Table 4). These resulted in an ICER of $\mathrm{S} \$ 33,833 / \mathrm{QALY}$ gained for TF-TAVI.

\section{Deterministic Sensitivity and Scenario Analyses}

Results of cost-effectiveness analysis from a number of one-way DSAs are shown in Figure 2. The model was the most sensitive to changes of operational costs for TF-TAVI and SAVR. However, the models were robust as ICERs remain similar when applying significant changes on parameters.

The results of scenario analyses are listed in Table 5. Shortening the time horizon while holding all other base-case assumptions constantly resulted in larger ICERs for TF-TAVI. Using the events rates based on the newer TAVI systems generated more favourable results for TF-TAVI. Assuming no new cases for long-term complications or the 2 treatments had same probabilities of stroke, MI, and AKI beyond 2 years also led to higher ICERs.

\section{Probabilistic Sensitivity Analysis}

The cost-effectiveness acceptability curve for TAVI and SAVR is presented in Figure 3. At a Willingness to Pay (WTP) threshold of $\$ \$ 73,167$ per QALY gained, the probability of TF-TAVI being cost-effective was $98.2 \%$.

Similarly, the cost-effectiveness plane with 100,000 incremental costs and QALYs based on the Monte Carlo simulations (Fig. 4) showed that TF-TAVI was economically attractive in $98.19 \%$ of replicates at the threshold. 
Table 4. Primary Cost-effectiveness Analysis for TF-TAVI versus SAVR

\begin{tabular}{lccccc}
\hline Option & Costs (S\$) & Incremental Costs (S\$) & Effectiveness (QALYs) & $\begin{array}{c}\text { Incremental Effectiveness } \\
\text { (QALYs) }\end{array}$ & ICER \\
\hline SAVR & 69,140 & & 3.67 & & $33,833 /$ \\
TF-TAVI & 75,386 & 5,852 & 3.86 & 0.21 & QALY \\
\hline
\end{tabular}

ICER: incremental cost-effectiveness ratio; QALYs: quality-adjusted life-years; SAVR: Surgical aortic valve replacement; TF-TAVI: Transfemoral transcatheter aortic valve replacement

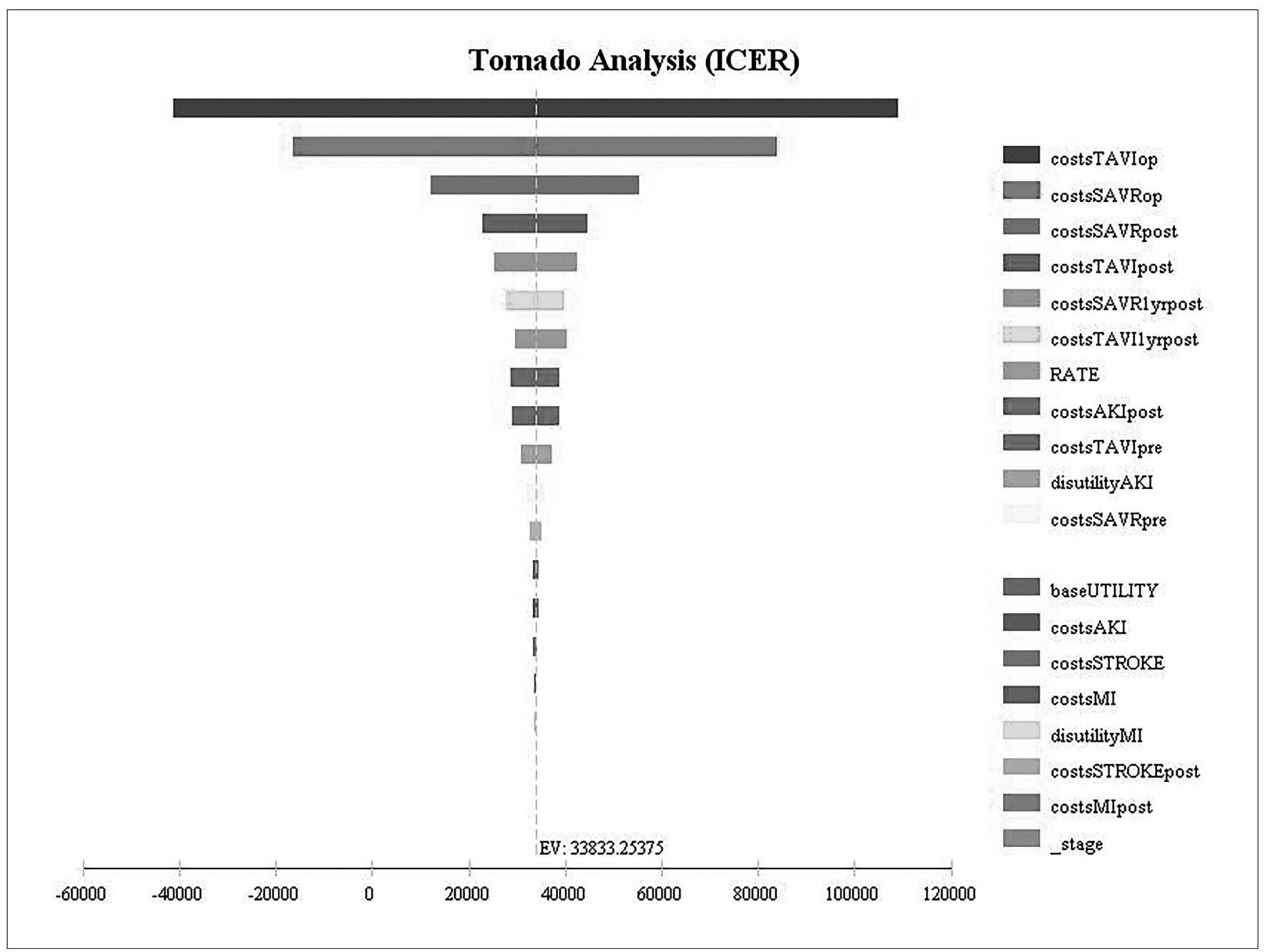

Fig. 2. One-way Sensitivity Analysis for TF-TAVI versus SAVR.

AKI: Acute kidney injury; ICER: Incremental cost-effectiveness ratio; MI: Myocardial infarction; SAVR: Surgical aortic valve replacement; TF-TAVI: Transfemoral transcatheter aortic valve replacement

costsTAVIop: Costs of TAVI operation; costsSAVRop: Costs of SAVR operation; costsSAVRpost: Post-procedural costs of SAVR; costsTAVIpost: Post-procedural costs of TAVI; costsSAVR1yrpost: One-year follow up costs of SAVR; costsTAVI1yrpost: One-year follow up costs of TAVI; RATE: Rate; costsAKIpost: Subsequent years costs of AKI; costsTAVIpre: Pre-procedural costs of TAVI; disutilityAKI: Disutility of AKI; costsSAVRpre: Pre-procedural costs of SAVR; baseUTILITY: Baseline utility; costsAKI: First year costs of AKI; costsSTROKE: First year costs of stroke; costsMI: First year costs of MI; disutilityMI: Disutility of MI; costsSTROKEpost: Subsequent years costs of stroke; costsMIpost: Subsequent years costs of MI; _stage: Stage 
Table 5. Scenario Cost-effectiveness Analysis for TF-TAVI versus SAVR

\begin{tabular}{lcc}
\hline & 8 years horizon & 5 years horizon \\
\hline Holding other base case assumptions constant* & $33,833 / \mathrm{QALY}$ & $60,825 / \mathrm{QALY}$ \\
\hline Using the event probabilities from the PARTNER 2 S3 propensity score analysis & $21,732 / \mathrm{QALY}$ & $44,598 / \mathrm{QALY}$ \\
\hline No new cases for stroke, MI, AKI beyond 2 years & $35,588 / \mathrm{QALY}$ & $61,096 / \mathrm{QALY}$ \\
\hline $\begin{array}{l}\text { Assuming the two treatments have the same probabilities of stroke, MI, AKI at 2 } \\
\text { years for the remaining years }\end{array}$ & $23,781 / \mathrm{QALY}$ & $59,863 / \mathrm{QALY}$ \\
\hline
\end{tabular}

AKI:Acute kidney injury; MI: Myocardial infarction; QALYs: Quality-adjusted life-years; SAVR: Surgical aortic valve replacement; TF-TAVI: Transfemoral transcatheter aortic valve implantation

*Base case analysis: apply life table mortality for both TAVI and SAVR, 8 years horizon, and assume probabilities of stroke, MI, and AKI of the 2 treatments after 2 years are same.

\section{Discussion}

To the best of our knowledge, this is the first study investigating the cost-effectiveness of TF-TAVI versus SAVR in intermediate and low risk patients with severe symptomatic AS using Singapore's local healthcare cost data. The model-based economic analysis indicated that TF-TAVI is most likely a cost-effective treatment when compared with SAVR, as it generated modestly higher QALY gains with slightly higher costs, resulting in an ICER much lower than the commonly used WTP threshold in Singapore (i.e. S\$73,167). Moreover, the findings were robust to various sensitivity analyses. As a result, TF-TAVI could be an economically attractive strategy in treating patients with severe symptomatic AS in Singapore.

Differences in costs and clinical outcomes determined the cost-effectiveness of TF-TAVI compared to

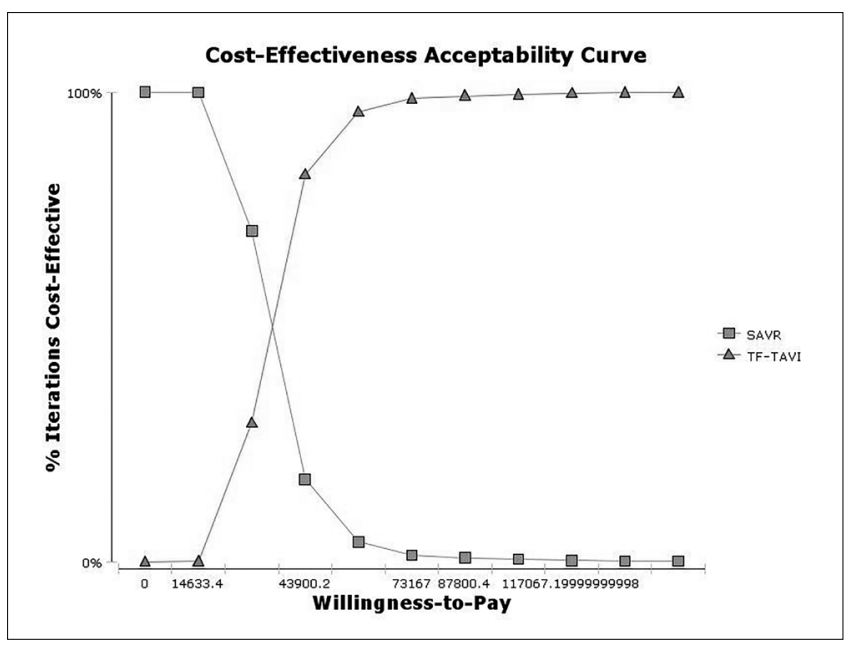

Fig. 3. Cost-effectiveness Acceptability Curve for TF-TAVI versus SAVR in Intermediate-Low Risk Patients.

SAVR: Surgical aortic valve replacement; TF-TAVI: Transfemoral transcatheter aortic valve replacement
SAVR. TF-TAVI had substantially greater costs than SAVR in terms of the device; while its less aggressive nature led to significantly lower costs in other aspects such as manpower, facility fees, and post-procedural costs. This trade-off resulted in TF-TAVI having a comparable hospital costs with SAVR (Table 2). This means that the differences in cost-effectiveness were mainly driven by clinical outcomes. The trial data had demonstrated that TF-TAVI had better or similar performance for the majority of short- and longterm outcomes in comparison with SAVR (Table 1), resulting in the divergence in cost-effectiveness outcomes between them.

Baron and colleagues have conducted cost analysis and assessed the cost-effectiveness of TAVI versus SAVR in patients with intermediate-risk for surgery from the PARTNER 2A randomised control trial (RCT)

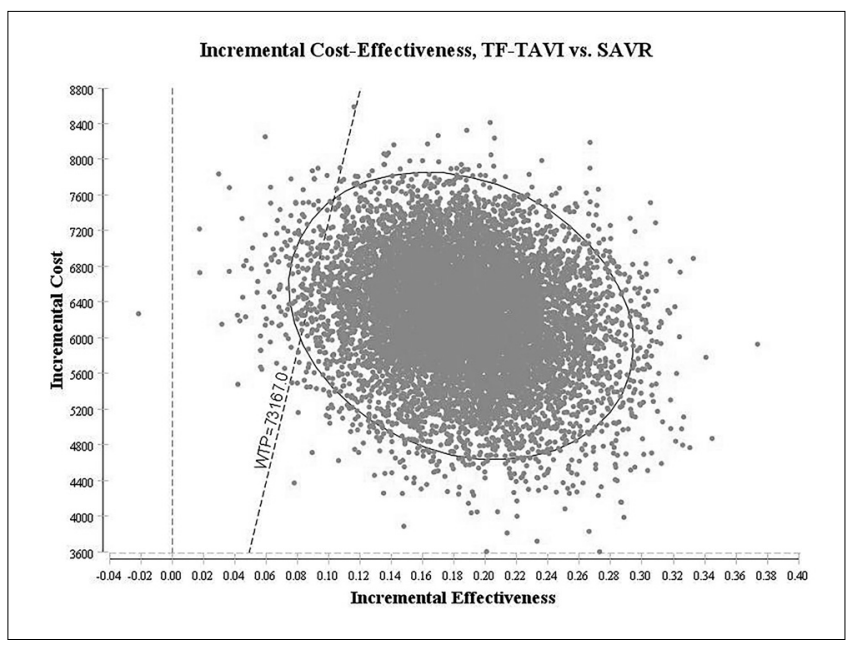

Fig. 4. Cost-effectiveness Plane of TF-TAVI versus SAVR.

SAVR: Surgical aortic valve replacement; TF-TAVI: Transfemoral transcatheter aortic valve replacement 
and S3i observational study. ${ }^{33}$ Index hospitalisation cost was US $\$ 18,000$ lower with SAPIEN XT TAVI or US $\$ 23,000$ lower with SAPIEN 3 than with SAVR mainly due to $4.5-5.6$ days shorter post-procedure hospital stay. Cumulative 2-year costs with SAPIEN XT TAVI (77\% TF access) system was US $\$ 6,414$ less than SAVR ( $\mathrm{p}<0.001)$, and cumulative 1-year costs with SAPIEN 3 TAVI (88\% TF access) was US $\$ 15,511$ less than SAVR. Cost savings in both cases were mainly due to fewer repeat hospitalisations, and less use of rehabilitation and skilled nursing facilities in the TAVI group. Using the in-trial EQ-5Q assessment results, the authors found TAVI to be economically dominant with either the SAPIEN XT or the SAPIEN 3 valve system when compared to SAVR. Difference in QALY gain with SAPIEN XT TAVI or SAPIEN 3 TAVI vs SAVR was 0.15 (95\% CI: $-0.08-0.39$ ) and 0.27 (95\% CI: $0.06-0.51$ ), respectively. Comparably, our results showed similar estimated QALY gain with TF-TAVI $(0.21)$ as that of SAPIEN 3 TAVI in the Baron 2018 study, which had $88 \%$ TF-TAVI patients. Our index hospitalization cost post-procedure is half of SAVR for TAVI patients, because average TAVI post-procedure hospital stay is half of that for SAVR patients. However, 1-year follow up costs are very low for both TAVI and SAVR patients in Singapore compared with that in the US. This reflects the differences between the 2 healthcare systems.

As TAVI cases have grown tremendously in the past 5 years, real world evidence becomes important to evidence-based practitioners. In the case of balloon-expandable TAVI systems, real world evidence appears to be better than RCTs. In intermediate-risk patients, PARTNER 2A reported all-cause mortality rate at 1 -year as $14.5 \%,{ }^{2}$ while PARTNER 2 S3 registry resulted in $7.4 \% .{ }^{34}$ Firstly, this may be due to TAVI being a relatively newer procedure compared to SAVR, and hence presents a learning curve for heart teams. Secondly, TAVI requires the collaboration of a multidisciplinary team including interventional cardiologists, cardiac surgeons, imaging cardiologists and radiologists. Patients were assessed with the Society of Thoracic Surgeons (STS) score, EuroScore II and frailty index before being determined eligible for TAVI. This ensures good patient selection and suitable candidates for the procedure translating to better procedural success. Lastly, PARTNER 2A TAVI cohorts received an older generation of TAVI valve and delivery system, while all patients in the PARTNER 2 S3 registry received the latest SAPIEN 3 valve system.
Technological advances may further contribute to better clinical results.

Despite the promising clinical results, access to TAVI has been limited in Singapore. To date more than 650 TAVI heart valves have been implanted since its first use in $2009^{35}$ which translates to around 25 valves per million population per year. In developed countries around the region such as Australia and Japan, the implant rates are estimated to be 100 and 55 per million population per year, respectively. The few factors contributing to the low adoption of TAVI treatment in Singapore are low patient disease awareness; low affordability in elderly patients; and lack of government reimbursement for such treatments. TAVI is reimbursed by national insurance or regional funding in developed countries across the world ${ }^{19}$ and many reimbursement decisions were based on providing better treatment to patients and the costeffectiveness of new therapy. The results of this study together with existing clinical results will provide important insights to policy makers in prioritizing TAVI treatment for our patients.

Our analysis has several limitations. Firstly, since long-term clinical outcome data is not yet available, a number of assumptions were made during the extrapolation procedure, which implies some degree of uncertainty of the results. However, the results of the scenario analyses indicated that either shortening the time horizon, or assuming treatment specific probabilities after the maximum available outcome duration, yielded the same results. Our results are thus robust to parameter changes in sensitivity and scenario analyses. Secondly, we used clinical results from PARTNER 2A and PARTNER $2 \mathrm{~S} 3$ trials other than Singapore local patient outcomes data in modelling the parameters. This is because Singapore has a relatively limited number of TAVI cases (compared to Europe and US) and we do not have any locally randomized trials. Although using overseas trial data is not ideal, using local clinical data is more problematic because of the significant selection bias. In clinical practice, open surgery will only be performed for patients who are assessed to be able to benefit from it, while TAVI is considered for severe patients who are not well enough for open surgery. As a result, clinical outcomes of open surgery and TAVI are incomparable and can lead to invalid conclusions if they are used to evaluate the relative merits of the 2 treatment strategies such as in economic evaluation. Therefore, overseas trial data has been used in many CEA studies comparing TAVI versus SAVR. ${ }^{9-14,33}$ 
Thirdly, factors such as re-hospitalisation, re-intervention, and concomitant coronary artery bypass surgery were not included in the analysis due to data unavailability. Those factors could affect the cost-effectiveness results. Similarly, there is no good way of quantifying the long term impact of PVL on health economic outcomes (mainly due to data unavailability), although we know that moderate PVL can lead to increased hospitalisation and mortality, and there was indeed more moderate PVL in the TAVR arm. Lastly, our findings were specific to the balloon-expandable SAPIEN systems only, and may not be generalised to other TAVI technologies, such as the self-expanding TAVI systems.

\section{Conclusion}

From the perspective of the Singapore healthcare system, TF-TAVI is highly likely to be a cost-effective treatment option for intermediate and low risk patients with severe symptomatic aortic stenosis, in comparison with SAVR. Our study would provide the first evidence for the potential of TAVI to be an alternative treatment to the conventional SAVR in Singapore. The positive result might contribute to the future formulation of local clinical practice guidelines or health policies. The favorable outcome of this study may encourage the Singapore Ministry of Health to implement policies to utilise this specific treatment for severe AS and intermediate surgical risk patients.

Funding: Singapore Ministry of Health, National Medical Research Council research grant CNIG14NOV009

\section{Acknowledgement:}

The authors would like to thank the Singapore Ministry of Health National Medical Research Council for supporting this research, and Dr Brett Doble for providing valuable input on the health economics model. We extend our gratitude to Dr Viva Yan Ma from Becton Dickinson Medical (Singapore) for her help on promoting the study.

\section{REFERENCES}

1. Kapadia SR, Leon MB, Makkar RR, Tuzcu EM, Svensson LG, Kodali S, et al; PARTNER trial investigators. 5-year outcomes of transcatheter aortic valve replacement compared with standard treatment for patients with inoperable aortic stenosis (PARTNER 1): a randomised controlled trial. Lancet 2015;385:2485 - 91.

2. Leon MB, Smith CR, Mack MJ, Makkar RR, Svensson LG, Kodali SK, et al; PARTNER 2 Investigators. Transcatheter or surgical aortic-valve replacement in intermediate-risk patients. N Engl J Med 2016;374:1609 - 20.
3. Mack MJ, Leon MB, Smith CR, Miller DC, Moses JW, Tuzcu EM, et al; PARTNER 1 trial investigators. 5-year outcomes of transcatheter aortic valve replacement or surgical aortic valve replacement for high surgical risk patients with aortic stenosis (PARTNER 1): a randomised controlled trial. Lancet 2015;385:2477-84.

4. Nishimura RA, Otto CM, Bonow RO, Carabello BA, Erwin JP, 3rd, Fleisher LA, et al. 2017 AHA/ACC Focused update of the 2014 AHA/ACC Guideline for the management of patients with valvular heart disease: a report of the American College of Cardiology/ American Heart Association task force on clinical practice guidelines. Circulation 2017;135:e1159-95.

5. Baumgartner H, Falk V, Bax JJ, De Bonis M, Hamm C, Holm PJ, et al; Group ESCSD. 2017 ESC/EACTS Guidelines for the management of valvular heart disease. Eur Heart J 2017;38:2739-91.

6. Gada H, Kapadia SR, Tuzcu EM, Svensson LG and Marwick TH. Markov model for selection of aortic valve replacement versus transcatheter aortic valve implantation (without replacement) in high-risk patients. Am J Cardiol 2012;109:1326-33.

7. Reynolds MR, Lei Y, Wang K, Chinnakondepalli K, Vilain KA, Magnuson EA, et al; CoreValve USHRPTI. Cost-effectiveness of transcatheter aortic valve replacement with a self-expanding prosthesis versus surgical aortic valve replacement. J Am Coll Cardiol 2016;67:29-38.

8. Reynolds MR, Magnuson EA, Lei Y, Wang K, Vilain K, Li H, et al; PARTNER investigators. Cost-effectiveness of transcatheter aortic valve replacement compared with surgical aortic valve replacement in high-risk patients with severe aortic stenosis: results of the PARTNER (Placement of Aortic Transcatheter Valves) trial (Cohort A). J Am Coll Cardiol. 2012;60:2683-92.

9. Fairbairn TA, Meads DM, Hulme C, Mather AN, Plein S, Blackman DJ, et al. The cost-effectiveness of transcatheter aortic valve implantation versus surgical aortic valve replacement in patients with severe aortic stenosis at high operative risk. Heart 2013;99:914-20.

10. Geisler BP, Huygens SA, Reardon MJ, Van Mieghem N, Kappetein AP, Osnabrugge RLJ, et al. Cost-effectiveness and projected survival of self-expanding transcatheter versus surgical aortic valve replacement for high risk patients in a European setting: A dutch analysis based on the CoreValve high risk trial. Structural Heart 2017;1:267-74.

11. Health Quality Ontario. Transcatheter aortic valve implantation for treatment of aortic valve stenosis: a health technology assessment. Ont Health Technol Assess Ser 2016;16:1-94.

12. Ribera A, Slof J, Andrea R, Falces C, Gutierrez E, Del ValleFernandez R, et al. Transfemoral transcatheter aortic valve replacement compared with surgical replacement in patients with severe aortic stenosis and comparable risk: cost-utility and its determinants. Int J Cardiol 2015;182:321-8.

13. Doble B, Blackhouse G, Goeree R and Xie F. Cost-effectiveness of the Edwards SAPIEN transcatheter heart valve compared with standard management and surgical aortic valve replacement in patients with severe symptomatic aortic stenosis: a Canadian perspective. J Thorac Cardiovasc Surg 2013;146:52-60.e3.

14. Neyt M, Van Brabandt $H$, Devriese $S$ and Van De Sande S. A cost-utility analysis of transcatheter aortic valve implantation in Belgium: focusing on a well-defined and identifiable population. BMJ Open 2012;2.

15. Aalaei-Andabili SH, Anderson RD, Bavry AA, Lee TC, Wayangankar S, Arnaoutakis GJ, et al. Transcatheter aortic valve replacement: efficiency and safety improvements with progressive experience and improved technology. Innovations (Phila) 2018;13:120-24. 
16. Toppen W, Johansen D, Sareh S, Fernandez J, Satou N, Patel KD, et al. Improved costs and outcomes with conscious sedation vs general anesthesia in TAVR patients: Time to wake up? PLoS One 2017; 12:e0173777.

17. Pilgrim T, Lee JKT, O’Sullivan CJ, Stortecky S, Ariotti S, Franzone A, et al. Early versus newer generation devices for transcatheter aortic valve implantation in routine clinical practice: a propensity score matched analysis. Open Heart 2018;5:e000695.

18. Shahian DM, Gleason TG, Shemin RJ, Carroll JD and Mack MJ. TAVR 2.0: Collaborating to measure, assure, and advance quality. Ann Thorac Surg. 2019;107:329-30.

19. Mylotte D, Osnabrugge RLJ, Windecker S, Lefevre T, de Jaegere $\mathrm{P}$, Jeger R, et al. Transcatheter aortic valve replacement in Europe: adoption trends and factors influencing device utilization. J Am Coll Cardiol 2013;62:210-19.

20. The World Bank. Current health expenditure ( $\%$ of GDP). Available at: https://data.worldbank.org/indicator/SH.XPD. CHEX.GD.ZS. Accessed on 27 July 2020.

21. Ministry of Health Singapore. Speech by Mr Gan Kim Yong, Minister for Health, at the National Medical Excellence Awards Ceremony 2017. Available at: https://www.moh.gov.sg/ content/moh_web/home/pressRoom/speeches_d/2017/speech-bymr-gan-kim-yong--minister-for-health--at-the-national-0.html. Accessed on 27 July 2020

22. The World Bank. GDP per capita (current US\$) - Singapore. Available at: https://data.worldbank.org/indicator/NY.GDP.PCAP. $\mathrm{CD}$ ?locations $=\mathrm{SG}$. Accessed on 27 July 2020 .

23. Smith CR, Leon MB, Mack MJ, Miller DC, Moses JW, Svensson LG, et al; PARTNER trial investigators. Transcatheter versus surgical aortic-valve replacement in high-risk patients. N Engl J Med 2011;364:2187-98.

24. Statistics Singapore. Complete life tables 2014-2015 for Singapore resident population. Available at: https://www.singstat.gov. $\mathrm{sg} /$ publications/population/complete-life-table. Accessed on July 27,2020

25. Makkar RR, Thourani VH, Mack MJ, Kodali SK, Kapadia S, Webb JG, et al; PARTNER 2 investigators. Five-year outcomes of transcatheter or surgical aortic-valve replacement. N Engl J Med 2020;382:799-809.

26. Bronnum-Hansen H, Davidsen M, Thorvaldsen P, Danish MSG. Long-term survival and causes of death after stroke. Stroke 2001;32:2131-6.

27. Smolina K, Wright FL, Rayner M, Goldacre MJ. Long-term survival and recurrence after acute myocardial infarction in England, 2004 to 2010. Circ Cardiovasc Qual Outcomes 2012;5:532-40.

28. Tam DY, Hughes A, Wijeysundera HC, Fremes SE. Cost and effectiveness: can't have one without the other. J Thorac Cardiovasc Surg 2018;156:1851-3.

29. Lin L, Teng M, Zhao YJ, Khoo AL, Seet RC, Yong QW, et al. Long-term cost-effectiveness of statin treatment for primary prevention of cardiovascular disease in the elderly. Cardiovasc Drugs Ther 2015;29:187-97.

30. Yang F, Lau T, Luo N. Cost-effectiveness of haemodialysis and peritoneal dialysis for patients with end-stage renal disease in Singapore. Nephrology (Carlton) 2016;21:669-77.

31. Abdin E, Subramaniam M, Vaingankar JA, Luo N, Chong SA. Population norms for the EQ-5D index scores using Singapore preference weights. Qual Life Res 2015;24:1545-53.

32. Wang Y, Xie F, Kong MC, Lee LH, Ng HJ, Ko Y. Cost-effectiveness of dabigatran and rivaroxaban compared with warfarin for stroke prevention in patients with atrial fibrillation. Cardiovasc Drugs Ther 2014;28:575-85.

33. Baron SJ, Wang J, House JA, Magnuson EA, Reynolds MR, Thourani VH, et al; PARTNER 2 Investigators. Cost-effectiveness of transcatheter vs surgical aortic valve replacement in patients with severe aortic stenosis at intermediate risk: results from the PARTNER 2 Trial. Circulation 2019;139:877-88.

34. Thourani VH, Suri RM, Gunter RL, Sheng S, O'Brien SM, Ailawadi G, et al. Contemporary real-world outcomes of surgical aortic valve replacement in 141,905 low-risk, intermediate-risk, and high-risk patients. Ann Thorac Surg 2015;99:55-61.

35. Edgar LW Tay. Transcatheter aortic valve implantation in Singapore: reflecting on the first decade. Ann Acad Med Singapore. 2020;49:271-2. 